\title{
Sensitivity estimation of LHAASO-WCDA for observing GLE events
}

\section{Yunfeng Zhang, Huanyu Jia* and Yonglin Feng}

School of Physical Science and Technology,Southwest Jiaotong University, Chengdu 610031, China E-mail: hyjia@swjtu.edu.cn

\begin{abstract}
Ground Level Enhancement (GLE) events of solar cosmic ray refer to the sudden, sharp and short-lived enhancement of ground level energetic particles generated from solar flare. The study of GLE events has been playing an important role in the study of solar activity and basic physics of cosmic rays. The Large High Altitude Air Shower Observatory (LHAASO), a multi-component instrument, is located at high altitude (4410 m a.s.1.) in Daocheng, Sichuan province, P.R. China, with the one of the main aims to observe GLE events. The sensitivity of LHAASO-WCDA to observe GLE events has been estimated in this paper. The minimum flux needed for LHAASO-WCDA to observe GLE event has been calculated by using the energy spectrum of 13 GLE events during 22 solar cycles. The result shows that LHAASO-WCDA can observe GLE events with the energy exceeds 50, 100, 500 or $1000 \mathrm{GeV}$.
\end{abstract}

Keywords: sensitivity, GLE, LHAASO-WCDA

$37^{\text {th }}$ International Cosmic Ray Conference (ICRC 2021)

July 12th - 23rd, 2021

Online-Berlin, Germany

${ }^{*}$ Presenter 


\section{Introduction}

Ground Level Enhancement (GLE) [1] events of solar cosmic ray refer to the sudden, sharp and short-lived enhancement of ground level energetic particles with energy above $450 \mathrm{MeV}$ generated from solar flare and coronal mass ejection (CME). Since Frobush [2] discovered GLE events, 72 GLE events have been recorded. The probability of occurrence of GLE events is higher during the maximum and declining phase of the solar activity cycle [3]. Solar activity will release an amount of high-energy particles (solar cosmic rays) during the GLE event. Among them, more than $90 \%$ are protons, followed by alpha particles, and a small number of electrons as well as other particles. The transport of these particles between the Sun and Earth just takes about 10 minutes, and usually cause adverse effect on aviation, space and communications. Therefore, the study of GLE events is not only an important sample to understand the mechanism of the generation, acceleration and propagation of cosmic rays, but also helpful to the prediction and early warning of catastrophic space weather.

The solar energetic particle detection during GLE events is mainly divided into direct and indirect detection. Direct detection usually refers to measure solar energetic particles by satellite experiments like ACE, STEREO, SOHO and GOES. As we well know that the observed capacity of the satellite is generally in energy ranges from $\mathrm{KeV}$ to a few hundred $\mathrm{MeV}$, so particles with higher energies can only be detected by ground based detector. Due to the existence of the earth atmosphere, the solar energetic particles can generate atmospheric shower by the cascade in the atmosphere [4]. Therefore, a large number of secondary particles will be produced, including hadrons, electrons, muons and photons etc. The secondary particles, measured by using ground based detector, can be used to infer the properties of primary solar energetic particles. Hence, based-ground detection is an indirect detection. Common ground detections, include neutron monitors and EAS array experiments, can detect solar energetic particles with energies above GeV. For instance, the IceTop [5] experiment detected solar energetic particles in the energy range of 0.6-7.6 GeV during the GLE70 event. It is also reported that the energy of the solar energetic particles is several hundred GeV. For example, the BUST [6] experiment detected the energy of solar energetic protons with energy above $500 \mathrm{GeV}$ during the GLE42 event. Theoretical prediction has reported that the upper limit of the energy of the solar cosmic rays released by solar activity is $3 \mathrm{TeV}$ [7]. According to the calculations [8], however, these particles could be accelerated to higher energies, $10 \mathrm{TeV}$. More observational results are needed to verify whether the energetic solar particles in GLE events can reach hundreds of $\mathrm{GeV}$, or even $\mathrm{TeV}$. The observation of GLE events is also one of the scientific aim in the Large High Altitude Air Shower Observatory (LHAASO). Whether the LHAASO experiment can observe high-energy GLE events, the first problem to be solved is the sensitivity estimation of GLE events observed by the LHAASO experiment. In this work, we estimate the sensitivity of the LHAASO-WCDA experiment to observe GLE events under different zenith angles, observation energies and GLE time durations by using proton observational peak energy spectrum of 13 GLE (GLE40-GLE52) events in 22 solar cycles. 


\section{Calculation method of sensitivity for observing GLE events in LHAASO-WCDA experiment}

The Large High Altitude Air Shower Observatory (LHAASO), an extensive air shower (EAS) array, is located at high altitude (4410 $\mathrm{m}$ a.s.l.) in Daocheng, Sichuan province. The array consists of a $1.3 \mathrm{~km}^{2}$ array (KM2A), a water Cherenkov detector array (WCDA), and 18 wide field-of-view air Cherenkov/fluorescence telescopes (WFCTA). Among them, LHAASO-WCDA, covering an area of $\sim 78,000 \mathrm{~m}^{2}$, is located at experiment center and constituted by 3120 detector units as well as divided into 3 separate arrays. More details of LHAASO-WCDA can be found elsewhere [9 10]. In LHAASO experiment, LHAASO-WCDA detection threshold can be as low as a few dozen GeV, which is possible for the LHAASO experiment to study solar cosmic rays. This means that GLE events can be observed.

The sensitivity of LHAASO-WCDA to observe GLE events refers to the minimum flux of GLE solar energetic particles that exceeds 5 times the standard deviation $(\sigma)$ of background count of LHAASO-WCDA (galactic cosmic rays, GCR). During the GLE event, the background count $N_{\mathrm{GCR}}$ can be calculated as follows:

$$
N_{\mathrm{GCR}}=T \cdot \iint_{E_{\min }}^{E_{\max }}\left(\frac{d N}{d E}\right)_{\mathrm{GCR}} \cdot A_{\mathrm{eff}}(E, \theta) \cdot d E \cdot d \Omega
$$

Here, $T$ is time duration of GLE, $E_{\max }$ is energy cutoff of GCR $\left(10^{20} \mathrm{eV}\right), A_{\mathrm{eff}}(E, \theta)$ is WCDA's effective area, $\left(\frac{d N}{d E}\right)_{\mathrm{GCR}}$ is the energy spectrum of $\mathrm{H}$ [11] add He [12] of GCR, $\Omega$ is solid angle ( $d \Omega=\sin \theta \cdot d \theta \cdot d \varphi, \theta$ is zenith angle, $\varphi$ is azimuth), $E_{\min }$ is the minimum observation energy of LHAASO-WCDA experiment. For zenith angle $0-15^{\circ}, 15-30^{\circ}, 30-45^{\circ}$ and $45-60^{\circ}$, the minimum observed energy is 50,50,60 and $127 \mathrm{GeV}$, respectively. According to the background count $N_{\mathrm{GCR}}$, the minimum count $N_{\mathrm{GLE}}$ required for $5 \sigma\left(\sigma=\sqrt{N_{\mathrm{GCR}}}\right)$ observation of GLE events can be calculated by

$$
N_{\mathrm{GLE}}=5 \cdot \sqrt{N_{\mathrm{GCR}}}
$$

It can also be expressed as

$$
N_{\mathrm{GLE}}=T \cdot \iint_{E_{\min }}^{E_{\max }^{\cdot}}\left(\frac{d N}{d E}\right)_{\mathrm{GLE}} \cdot A_{\mathrm{eff}}(E, \theta) \cdot d E \cdot d \Omega
$$

Here, $E_{\max }^{\cdot}$ is energy cutoff of solar energetic particles in GLE events $\left(E_{\max }^{\cdot}=10 \mathrm{TeV}\right) .\left(\frac{d N}{d E}\right)_{\mathrm{GLE}}$ is energy spectrum of GLE events and can be expressed as $K E^{-\alpha}$, where $K$ is the normalization constant and $\alpha$ is the power law index. The normalization constant can be calculated from equations (3) when the specific value of the relevant variable $\left(\theta, T, E_{\min }, E_{\max }^{\cdot}, \alpha, A_{\mathrm{eff}}(E, \theta), N_{\mathrm{GLE}}\right)$ is given. The sensitivity $F$ is the minimum flux $(5 \sigma)$ required by LHAASO-WCDA to observe GLE events. It can be calculated by $K$ and equations (4).

$$
F=T \cdot \iint_{E_{\min }}^{E_{\max }} K \cdot E^{-\alpha} \cdot d E \cdot d \Omega
$$




\section{The calculation results and discussion of the sensitivity of LHAASO-WCDA in observing GLE events}

The GLE events can be observed by LAASO-WCDA when the solar energetic particle flux $I$ in GLE event is higher than $F$. The solar energetic particle flux $I$ of GLE events can be calculated by

$$
I=T \cdot \iint_{E_{\min }}^{E_{\max }^{\cdot}} I_{0} \cdot E^{-\beta} \cdot d E \cdot d \Omega
$$

Here, $I_{0}$ is energy spectrum coefficient of GLE events, $\beta$ is energy spectrum index. By extrapolating proton average differential peak energy spectra $\left(I_{0} \cdot E^{-\beta}=(2.61 \pm 0.25) \cdot 10^{6} \cdot E^{-(3.32 \pm 0.08)}\left[\mathrm{m}^{-2}\right.\right.$ $\mathrm{s}^{-1} \mathrm{sr}^{-1} \mathrm{GeV}^{-1}$ ]) of $13 \mathrm{GLE}$ events in 22 solar cycle at energy range from 3 to $10 \mathrm{GeV}$ [13], the average peak flux $I$ of solar energetic particles can be given. At the same time, the sensitivity $F$ can be calculated by using the WCDA's effective area [14] and proton peak energy spectrum index (-3.32) of 13 GLE events in 22 solar cycle. So the ability of LHAASO-WCDA to observe GLE events can be examined with comparing the calculated results of $I$ and $F$.

For high energy particles with different zenith angles, the effective areas of WCDA are different. Figure 1 shows the sensitivity $F$ of LHAASO-WCDA to observe GLE events (green triangle) and the flux $I$ of solar energetic particles (blue dot) were calculated over different zenith angle ranges $\left(0-15^{\circ}, 15-30^{\circ}, 30-45^{\circ}, 45-60^{\circ}\right)$ when the lower limit of WCDA observation energy is 127 $\mathrm{GeV}$. Also, the $I / F$ (red square) with different zenith angles is plotted. These results show that the sensitivity of LHAASO-WCDA to observe GLE events becomes terrible and $I / F$ decreases with increasing zenith angle. When the zenith angle is up to $45-60^{\circ}$, however, the $I / F$ is still greater than 1. So LHAASO-WCDA can also detect GLE events over larger zenith angle ranges.

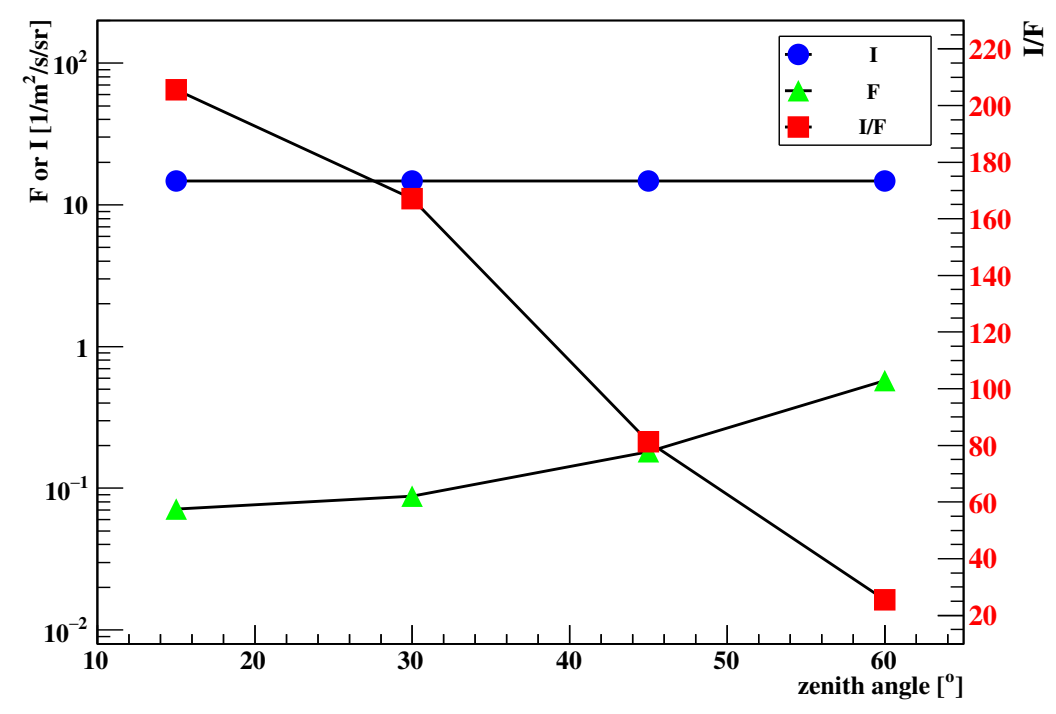

Fig. 1. The solar energetic particle flux, sensitivity and its ratio as a fluctuation of zenith angle.

At the same zenith angle, the energy of solar energetic particles and the time duration of GLE are also important factors to affect the ability of LHAASO-WCDA to detect GLE events. Figure 2 shows $F, I$ and $I / F$ as a function of the observed energy when the zenith angle is $0-15^{\circ}$. It can be 
seen that the ability of LHAASO-WCDA to detect GLE events decreases with the increase of the observed energy. But when the observed energy is more than $1 \mathrm{TeV}, I$ is 30 times than $F$, or even higher. This means that LHAASO-WCDA has a very strong ability to observe GLE particles with energy higher than TeV. In addition, dependence of $F, I$ and $I / F$ on the time duration are shown in figure 3 when the zenith angle is $0-15^{\circ}$ and minimum observed energy is $50 \mathrm{GeV}$. It can be seen that the possibility of LHAASO-WCDA to observe GLE events become larger with time duration.

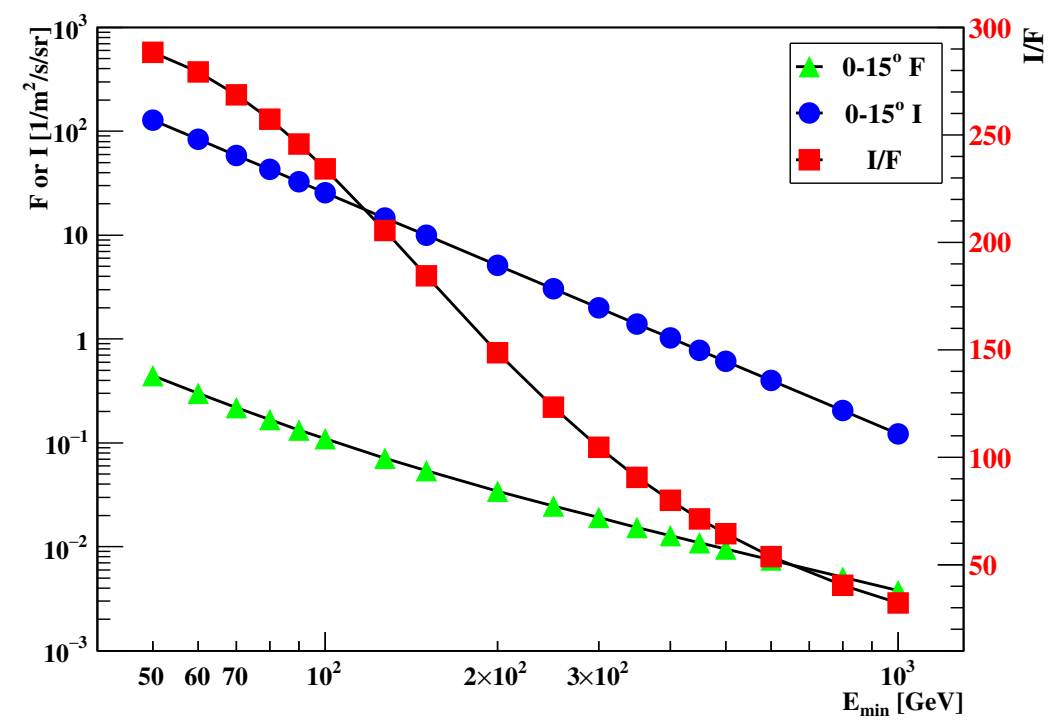

Fig. 2. The solar energetic particle flux, sensitivity and its ratio as a fluctuation of observation energy.

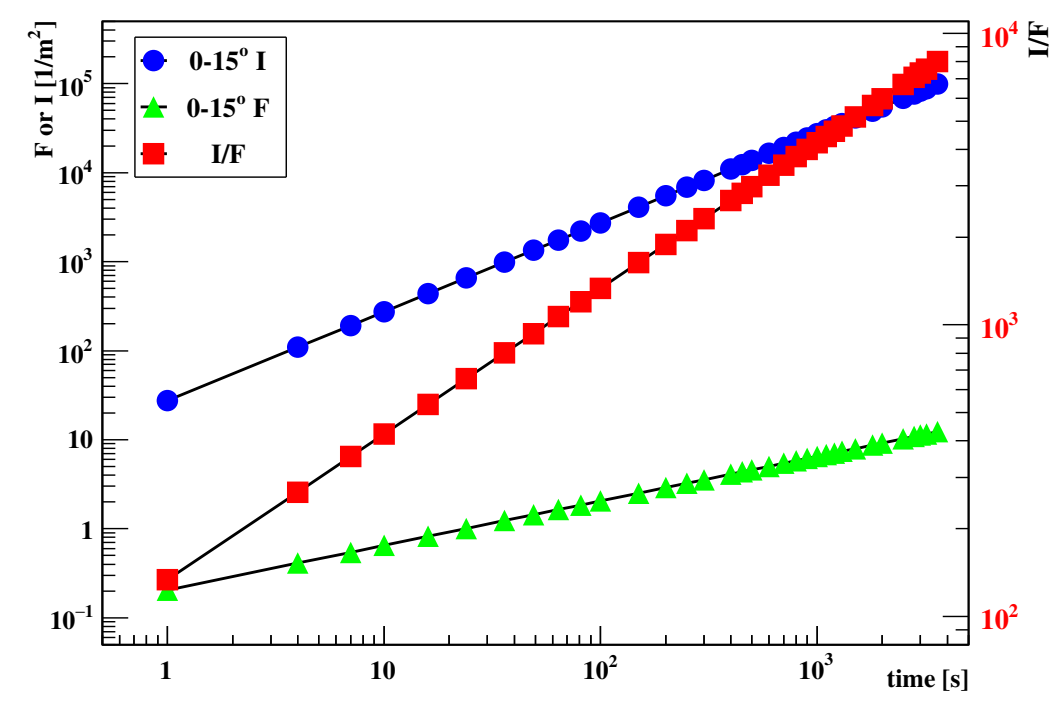

Fig. 3. The solar energetic particle flux, sensitivity and its ratio as a fluctuation of time duration. 


\section{Conclusions}

By using LHAASO-WCDA sensitivity calculation method for Observing GLE Events and combined with the observation results of 13 GLE events in solar cycle 22, the ability of LHAASOWCDA to observe GLE events for different zenith angles, observed energies and GLE time duration were analyzed in this paper. The results show that LHAASO-WCDA can observe GLE events with energy higher than $1 \mathrm{TeV}$ at zenith angle of $0-15^{\circ}$. It is worth noting that our results are obtained by extrapolation of the energy spectrum of GLE events, this means that the energy spectrum of the solar energetic particles remains unchanged at higher energies.

\section{Acknowledgments}

This paper is supported by the National Natural Key R\&D Program of China (Grant No. 2018YFA0404202) and the National Natural Science Foundation of China (Grant No.11947404).

\section{References}

[1] J. A. Simpson, Astrophysical Phenomena Discovered by Cosmic Ray and Solar Flare Ground Level Events: The Early Years, in Proceedings of 21th ICRC, 12 (1990), pp187-195.

[2] S. E. Forbush, Three Unusual Cosmic-Ray Increases Possibly Due to Charged Particles from the Sun, Phys. Rev., 70 (1946), 771-772.

[3] M. A. Shea et al., A Summary of Major Solar Proton Events, Sol. Phys., 127 (1990), 297-320.

[4] P. Auger et al., Extensive Cosmic-Ray Showers, Rev. Mod. Phys., 11 (1939), 288-291.

[5] R. Abbasi et al., Solar Energetic Particle Spectrum on 2006 December 13 Determined by IceTop, Astrophys. J., 689 (2008), L65-L68.

[6] S. N. Karpov et al., Muon bursts at the Baksan Underground Scintillation Telescope during energetic solar phenomena, Nuovo Cimento C, 21C (1998), 551-573.

[7] B. N. Srivastava, Basic nuclear physics and cosmic rays, 1979.

[8] L. Zhang et al., Time-Dependent Shock Wave Acceleration of Solar Flare's High Energy Particle: I. Basic Theory, in Proceedings of 23th ICRC, 3 (1993), pp33-36.

[9] Z. Cao for the LHAASO collaboration, A future project at tibet: the large high altitude air shower observatory (LHAASO), Chinese Phys. c, 34 (2010), 249-252.

[10] Z. Cao et al., Introduction to Large High Altitude Air Shower Observatory (LHAASO), Acta Astronomica Sinica, 60 (2019), 19.

[11] M. Aguilar for AMS Collaboration, Precision Measurement of the Proton Flux in Primary Cosmic Rays from Rigidity 1 GV to 1.8 TV with the Alpha Magnetic Spectrometer on the International Space Station, Phys. Rev. Lett., 114 (2015), 171103. 
[12] M. Aguilar for AMS Collaboration, Precision Measurement of the Helium Flux in Primary Cosmic Rays of Rigidities 1.9 GV to $3 \mathrm{TV}$ with the Alpha Magnetic Spectrometer on the International Space Station, Phys. Rev. Lett., 115 (2015), 211101.

[13] D. Mottl et al., The issues of reliability of solar energetic proton flux databases and models, Adv. Space Res., 39 (2007), 1355-1361.

[14] X. Bai et al., The Large High Altitude Air Shower Observatory (LHAASO) Science White Paper, [arXiv:1905.02773v1 [astro-ph.HE]], 2019. 